\title{
An integrated image analysis platform to quantify signal transduction in single cells
}

Pelet, Serge ; Dechant, Reinhard ; Lee, Sung Sik ; van Drogen, Frank ; Peter, Matthias

\begin{abstract}
Microscopy can provide invaluable information about biological processes at the single cell level. It remains a challenge, however, to extract quantitative information from these types of datasets. We have developed an image analysis platform named YeastQuant to simplify data extraction by offering an integrated method to turn time-lapse movies into single cell measurements. This platform is based on a database with a graphical user interface where the users can describe their experiments. The database is connected to the engineering software Matlab, which allows extracting the desired information by automatically segmenting and quantifying the microscopy images. We implemented three different segmentation methods that recognize individual cells under different conditions, and integrated image analysis protocols that allow measuring and analyzing distinct cellular readouts. To illustrate the power and versatility of YeastQuant, we investigated dynamic signal transduction processes in yeast. First, we quantified the expression of fluorescent reporters induced by osmotic stress to study noise in gene expression. Second, we analyzed the dynamic relocation of endogenous proteins from the cytoplasm to the cell nucleus, which provides a fast measure of pathway activity. These examples demonstrate that YeastQuant provides a versatile and expandable database and an experimental framework that improves image analysis and quantification of diverse microscopy-based readouts. Such dynamic single cell measurements are highly needed to establish mathematical models of signal transduction pathways.
\end{abstract}

DOI: https://doi.org/10.1039/c2ib20139a

Posted at the Zurich Open Repository and Archive, University of Zurich ZORA URL: https://doi.org/10.5167/uzh-79077

Journal Article

Originally published at:

Pelet, Serge; Dechant, Reinhard; Lee, Sung Sik; van Drogen, Frank; Peter, Matthias (2012). An integrated image analysis platform to quantify signal transduction in single cells. Integrative Biology, 4:1274-82.

DOI: https://doi.org/10.1039/c2ib20139a 


\title{
An integrated image analysis platform to quantify signal transduction in single cells
}

\author{
Serge Pelet ${ }^{*}$, Reinhard Dechant, Sung Sik Lee, Frank van Drogen and Matthias Peter* \\ Received (in XXX, XXX) Xth XXXXXXXXX 20XX, Accepted Xth XXXXXXXXX 20XX \\ s DOI: 10.1039/b000000x
}

\begin{abstract}
Microscopy can provide invaluable information about biological processes at the single cell level. It remains a challenge, however, to extract quantitative information from these types of datasets. We have developed an image analysis platform named YeastQuant to simplify data extraction by offering an integrated method to turn time-lapse movies into single cell measurements. This platform is based on a 10 database with a graphical user interface where the users can describe their experiments. The database is connected to the engineering software Matlab, which allows extracting the desired information by automatically segmenting and quantifying the microscopy images. We implemented three different segmentation methods that recognize individual cells under different conditions, and integrated image analysis protocols that allow measuring and analyzing distinct cellular readouts. To illustrate the power

15 and versatility of YeastQuant, we investigated dynamic signal transduction processes in yeast. First, we quantified the expression of fluorescent reporters induced by osmotic stress to study noise in gene expression. Second, we analyzed the dynamic relocation of endogenous proteins from the cytoplasm to the cell nucleus, which provide a fast measure of pathway activity. These examples demonstrate that YeastQuant provides a versatile and expandable database and experimental framework that improves

20 image analysis and quantification of diverse microscopy-based readouts. Such dynamic single cell measurements are highly needed to establish mathematical models of signal transduction pathways.
\end{abstract}

\section{Introduction}

${ }_{25}$ Cells are able to sense changes in their environment and deliver an appropriate response by integrating intra- and extra-cellular cues. Decades of biochemical analysis of signal transduction pathways have laid out the maps of signalling networks by identifying the major components involved in transmission of the 30 information. However, full understanding of signalling networks requires quantitative dynamic measurements of signal transmission in time and space, and the elucidation of positive and negative feedback regulation. Only such quantitative data will allow characterization of mechanisms that exhibit minor or 35 transient phenotypes, and the building of detailed mathematical models that comprehensively describe the signalling processes. Because population measurements often mask important regulatory mechanisms, quantitative single cell measurements are needed, as demonstrated more than ten years ago by Ferrel and ${ }_{40}$ Machleder ${ }^{1}$. This landmark study relied on biochemical methods to probe pathway activity upon hormone treatment in single oocytes, an approach that is unfortunately not possible for most cell types due to their smaller size.
Microscopy techniques, however, have been used since the 45 advent of biology to observe individual cells. While in the beginning these instruments allowed mostly a qualitative description of the specimen, subsequent technical improvements in detectors and light sources have transformed microscopy into a quantitative measurement technique. Moreover, the discovery of ${ }_{50}$ the green fluorescent proteins (GFP) provided the opportunity to follow the expression and localization of key proteins in living cells ${ }^{2}$. Multiple fluorescent protein spectral variants (e.g. cyan: CFP, yellow: YFP, red: RFP or mCherry) ${ }^{3}$ have since then been generated, and offer the opportunity to correlate the activity of 5 multiple proteins within a given cell. The linear relationship between fluorescence intensity and protein level also allows extracting relative protein concentrations from microscopy images. Recent dynamics experiments have demonstrated the power of this technique to understand complex regulatory 60 mechanisms in signal transduction in yeast and mammalian cells 4-8.

One major hurdle for the widespread use of microscopy for quantitative measurements is the difficulty in extracting information from images. While the human eye is well-trained to ${ }_{65}$ recognize objects within an image, this remains a complex task 
for a computer ${ }^{9}$. The microscopy image is a two dimensional matrix of pixel intensities. The segmentation process consists in identifying groups of pixels that belong to the same object (a cell or a nucleus, for instance) thereby allowing characterization of 5 various features of this object, such as size, shape or average pixel intensity.

Multiple tools have been developed to perform such tasks ${ }^{10,11}$, but an integration of the different segmentation methods, automated data extraction, annotation and storage in a single ${ }_{10}$ database is lacking. Here we present a new image analysis platform dedicated to the quantification of time-lapse microscopy data named YeastQuant. This versatile tool is based on a Filemaker database, which provides an intuitive interface for the user to enter a description of the experiment and keeps a record of 15 all experiments. This database is linked to the commonly used engineering software Matlab, which performs the required image analysis specified in the database. The output of the analysis can easily be visualized using dedicated Matlab programs with a simple user interface, exported to Excel, or further analysed by

${ }_{20}$ dedicated scripts. We demonstrate the power of this image analysis platform for studying signal transduction pathways in single cells by quantifying the expression and dynamic relocalization of different fluorescently tagged reporters.

\section{Results and Discussion}

\section{${ }_{25}$ YeastQuant platform}

The YeastQuant platform is fed with microscopy data sets generated by common microscopy software such as micromanager ${ }^{12}$, Metamorph (Molecular Devices) or TIF series (Figure 1). Typically images are acquired for different fields of view (set 30 of $\mathrm{X}$ and $\mathrm{Y}$ stage coordinates) at numerous time-points with multiple illumination settings. These images form a 4D data set.

The relevant information about the acquired data is then entered in the database (Filemaker) to describe the experiment. The database consists of three tables containing the experimental 35 description, the analysis flow and the segmentation parameters (Suppl. Figures 1, 2 and 3). The experimental description table contains information such as the sample type, the location and naming of the image files, the illumination settings, the magnification and binning used, the XY position description and

40 the type of image analysis to be performed on the dataset. While this description allows linking a given experiment to an analysis routine, the table describing the analysis flow specifies which images (based on the illumination settings entered previously) will be loaded, how the images are segmented, and allows the ${ }_{45}$ definition and measurements of different objects. The segmentation parameter table defines the general size of the objects to be found for a given analysis and thereby depends both on the analysis type and the magnification specified in the experiment record.

50 The Filemaker database is linked to the Matlab software via the standard JDBC protocol. Data analysis is directly performed using a dedicated script that automatically launches Matlab. Alternatively, by specifying the experiment number or another specific description of the experimental records, the parameters 55 of the experiments are queried together with the associated analysis description and segmentation parameters to generate a
Matlab structure. This structure contains all parameters required to perform the analysis, therefore the corresponding image files can be located and processed for the analysis.

${ }_{60}$ The Matlab analysis program is based on a set of small routines performing dedicated tasks such as image loading, image segmentation, object tracking, secondary object definition, image correction, object features measurement, measurement display and export (Suppl. Tables 1 and 2). The input and output ${ }_{65}$ parameters for these routines are defined in the analysis database. For example, for an image segmentation routine, the analysis database provides the name of the image to be used as an input (CFPImg) and the name of the final segmented object (Nucleus). A subsequent routine for object measurement can use for 70 example the "Nucleus" as input object along with another intensity image (YFPImg) to quantify the YFP fluorescent intensity in the nucleus. All the parameters required for the analysis are stored together with the file path of the images and measurements in the structure that is passed as input argument 75 and produced as output argument of each routine. Due to this simplicity and modularity, it is relatively straightforward to add additional routines to the main program.

To analyse the complete four-dimensional microscopy data set, the analysis is performed successively for each time-point for a 80 given field of view. This allows tracking objects from one frame to the next in the entire time-lapse movie, thereby obtaining a complete measurement set for a given object at all time points. However, because the analysis of different fields of view are independent from each other, these processes can easily be split ${ }_{85}$ and analysed in a parallel fashion on multiple CPU cores of a given machine. Using the parallel computing toolbox in Matlab, up to 12 threads can be simultaneously processed. More complex parallel architecture can also be established using the Matlab distributed computing server, if necessary.

Segmentation types

The more complex part of the analysis is to extract the contours of all cells from the intensity variations in the image. We have implemented four types of segmentation procedures depending 95 on the sample measured. The advantages and disadvantages of different segmentation methods are summarized in Table 1 . The code for the segmentation process is based on CellProfiler ${ }^{10}$, which was modified to the specificities of the image sets, and the employed Matlab routines are summarized in Suppl. Table 2.

100 The simplest segmentation code uses a fluorescent cytoplasmic marker to define the border of the cell. After setting the appropriate intensity threshold, the object pixels in the image can be identified. A water-shedding algorithm ${ }^{13}$ was further used to split individual cells (Figure 2A). The second segmentation 105 method uses an opposite approach by adding a fluorescent dye to the growth media surrounding the cells, and thus pixels with intensity below a given threshold were associated with cells (Figure 2B). This segmentation is limited by the thickness of the media layer, but it can be applied successfully in microfluidic 110 devices with a thickness up to 100 micrometres (Suppl. Figure 4). While these two methods identify the overall shape of the cell, it is also possible to visualize sub-cellular structures with appropriate fluorescent markers. To this end, we have developed a segmentation routine to quantify the nuclear and cytoplasmic ${ }_{115}$ pool of a given protein. The nuclei of individual cells were 
visualized by expressing fluorescently tagged histone Hta2, and a second fluorescent marker was then used to associate each nucleus with the cytoplasm of the corresponding cell (Figure 2C). Finally, we also developed a segmentation technique based on 5 bright field images. This segmentation method often becomes the method of choice if one of the following restrictions applies to the experimental measurements. First, if the fluorescent intensity changes during the time course of the experiment, thereby preventing proper detection of the cells in some frames of the 10 movie. Second, if large variability in fluorescence signals between cells cause problems, because the algorithms might bias the object recognition towards higher expressing cells and thereby misrepresent the diversity in fluorescence intensity present in the cell population. Third, if photo-toxicity caused by 15 the bright excitation light required for fluorescence imaging interferes with the cellular response. As shown in Figure 2D, two images were used for object recognition, one of them being slightly out of focus to enhance the contrast of the cell border. Using an edge detection algorithm, it was possible to identify 20 clusters of cells touching each other, and subsequently identify individual cells within these clusters using a Hough transform to recognize round objects ${ }^{14}$. The routines implementing these four different segmentation processes combined with object tracking (Suppl. Table 2) allows robust identification of individual yeast ${ }_{25}$ cells in a large set of microscopy time-lapse movies.

\section{Tracking}

Reliable identification of cells in the images is essential for proper tracking of individual cells. Using the bright field and 30 media segmentations we measured $96 \%$ efficiency in object recognition and $98 \%$ for the nuclear/cytoplasmic segmentation of cells, which have a nucleus in the focal plane of the image. These numbers depend on the quality of the images and on the cell density in the image. It is typically easier to recognize single cells 35 or groups of two to four cells compared to cell clusters composed of ten or more cell.

The tracking algorithm matches the positions of the centre of the objects in the current frame of the movie versus all the centres in the previous frame. The object with the closest distance to an

40 object in the previous frame (within a certain range) is assigned the tracking label of that object. If no matching object is found in the previous frame, the program will compare all earlier frames of the movie to find any object at that given position that can be matched to the current object.

45 The efficiency of the tracking in movies depends on many experimental factors. In the media segmentation experiment, $88 \%$ of the cells found in the first frame were tracked until the end. This percentage droped to $79 \%$ when using bright field segmentation, probably because in this experiment the seeding ${ }_{50}$ density of cells was higher and the time interval between each frame was larger, thus extending the total duration of the movie. In the nuclear/cytoplasmic segmentation experiments, this percentage was reduced to $67 \%$ although the fidelity of the cell segmentation was higher. This unexpected finding most likely 55 results from the fact that the nucleus can move within the cell and thus disappear from the image by leaving the focal plane.

Most of the performed experiments are of relatively short duration: ten minutes to a few hours, which allows less then three divisions. The daughter cells appear as new cells in the analysis
${ }_{60}$ once they reach a given size, however no information about the parental linage has been encoded in the program. For longer experiments, the dividing cells can quickly fill the whole field of view, making it very hard to segment and track individual cells. More importantly, daughter cells often grow out of the focal ${ }_{65}$ plane of the microscope preventing their proper detection. This situation can be improved by using microfluidic devices with a height matching the size of the yeast cells, which restricts cell division to a single plane and has been used efficiently to track cells over many generations ${ }^{15,16}$.

70

\section{Secondary Objects}

Although the primary segmentation method provides one or two objects, it is often necessary to define other regions within or around these objects. As shown in Figure 2E, such secondary 75 objects can either be defined by geometrical parameters or deduced from intensity information from an additional image. For example, the secondary object highlighted in red and called "media" was made of pixels outside of the cell, which are more than 2 but less than 10 pixels away from the border of the 80 primary cell object. This secondary object could equally well be defined inside the cell with slightly different parameters to quantify for instance the intensity of the cell membrane. The secondary object in blue was based on the intensity of a fluorescent image, of which $30 \%$ of all pixels with the highest ${ }_{85}$ intensity within the primary object were selected. This threshold allows exclusion of low intensity pixels, which often belong to the edge of the cell or belong to the large vacuole present in yeast cells, thus allowing concentrating the analysis on cytoplasmic and nuclear signals.

90 Taken together, the described segmentation methods allow the automatic identification of cells in images and combined with secondary objects, the region of measurement can be further refined. The ability to identify and track individual cells in timelapse movies allows dynamic quantification of the transduction of ${ }_{95}$ information, and thereby provides the experimental basis to study signalling networks and cellular outputs at the single cell level.

\section{Protein expression}

Quantifying the expression of transcriptional targets is a commonly used readout to quantify pathway activity upon 100 stimulation, and can be achieved with GFP-tagged proteins. However, these measurements are often limited by low expression levels or by interference of the large GFP moiety with the function of the endogenous protein. Moreover, the expression levels of endogenous protein reporters are dependent on 105 additional regulatory mechanisms, such as protein translation or protein stability. To circumvent these problems, synthetic expression reporters have been generated which are based on selected promoters directly driving the expression of $\mathrm{GFP}^{4,6}$. To improve the signal to noise ratio of these assays, we fused 110 multiple fluorescent proteins together generating quadrupleVenus $(\mathrm{qV})$ or quadrupleCFP (qCFP) expression reporters. For example, to study the expression induced upon activation of the high osmolarity glycerol (HOG) MAP-kinase signalling pathway, we placed these reporters under the control of the STL1-promoter, a 115 well-characterized transcriptional target of the HOG-pathway in budding yeast (Figure 3 ). Both reporters were integrated at two different loci, thus allowing analysis of pathway activation in 
single cells and quantification of the noise in the expression process. Images were acquired every 10 minutes for 2 hours at 5 different stage positions in three different wells. At each stage position, CFP and YFP images were recorded along with two 5 bright-field images. To quantify the dynamics of expression of these reporters, we relied on the bright field segmentation, which uncouples the detection of the objects from the fluorescence intensities present in the cells. This allowed tracking and quantification of 724 cells from the beginning to the end of the 10 movie, which corresponds to almost 50 cells per field of view. Upon treatment with $0.1,0.15$ and $0.2 \mathrm{M} \mathrm{NaCl}$, an increase in CFP and YFP fluorescence was detected 30 min after induction, and the fluorescence signal plateaued two hours after induction (Figure 3A and Suppl. Figure 5A). The standard deviation 15 displayed on these curves revealed a large cell-to-cell variability in reporter induction, demonstrating a large heterogeneity in a given population.

To identify the source of this noise, we used a strategy devised by Elowitz and co-workers ${ }^{17}$, which correlates the expression of the

20 two fluorescent reporters in the same cell allowing the quantification of cell-to-cell (extrinsic) and the within-cell (intrinsic) noise. A scatter plot was used to display the change in CFP vs. YFP intensity for all cells (Figure $3 \mathrm{~B}$ and Suppl. Figure $5 \mathrm{~B}$ ), and demonstrated the lack of correlation between the 25 expression of the $\mathrm{qV}$ and $\mathrm{qCFP}$ reporter constructs and the strong contribution of intrinsic noise in this system (Suppl. Figure 5C). Four cells were selected (highlighted with coloured circles), and single cell traces (Figure 3C and D) and intensity images (Suppl. Figure 5D) illustrate their different expression behaviours. These ${ }_{30}$ results demonstrate that the reporters can be expressed independently of each other, and provided important new insight into the cellular mechanisms governing expression noise ${ }^{6}$.

While establishing protein expression assays is relatively straightforward for most signalling cascades, they suffer from an

35 important drawback: as shown in Figure $3 \mathrm{~A}$, the increase in fluorescence intensity was detected only $30 \mathrm{~min}$ after induction of the pathway (time 0), when HOG pathway activity has already returned to basal levels and transcription has stopped ${ }^{18}$. This long lag phase mostly arises from the slow maturation time of the 40 fluorescent proteins ${ }^{19}$, and unfortunately prevents observing fast dynamics of signal transduction with this type of assay. Moreover, due to the long half-life of the expressed fluorescent protein, only increases in transcriptional activity can be measured without further modifications of the assay.

45

\section{Dynamic relocation}

Activation-dependent localization changes of signalling proteins provide powerful reporter assays to quantify signalling networks. Because the fluorescent protein is already expressed at the time of s0 pathway activation, the dynamics are only limited by the active or passive transport of the protein between the different cellular compartments. A recurring behaviour in many signalling pathways is the relocation of cytoplasmic proteins to the cell nucleus ${ }^{20}$. Yeast cells have a diameter of roughly $5 \mu \mathrm{m}$, and 55 relocalization from the plasma membrane to the nucleus via diffusion happens in less than 30 seconds ${ }^{6}$. Another example is the relocation of the MAP-kinase scaffold Ste5 to the plasma membrane which has been quantified by microscopy and occurs in less than 10 seconds ${ }^{21}$. The high temporal and spatial 60 resolution provided by fluorescence microscopy allows studying signal transduction kinetics in real time.

As an illustration, we constructed a strain that allows correlating the activity of the general stress transcription factor Msn2 and the MAP kinase Hog1. Hog1 rapidly translocates to the nucleus upon ${ }_{65}$ activation of the pathway by hyperosmotic shock ${ }^{22,23}$. Msn2 is cytoplasmic under normal growth conditions and accumulates in the nucleus upon various kinds of stresses such as glucose starvation, osmotic shock or temperature shifts ${ }^{24,25}$. This latter relocation event is triggered by multiple phosphorylation events 70 on Msn2, and detailed analysis allowed generation of a truncated construct that specifically responds to glucose starvation (Msn2NLS-GFP) ${ }^{25}$. To investigate the role of $\mathrm{Hog} 1$ in the activation of the general stress response, a strain expressing Hog 1 tagged with mCherry was transformed with plasmids harbouring either full75 length Msn2-GFP (Msn2-FL-GFP) or the Msn2-NLS-GFP fragment, and the two strains were mixed at equivalent concentrations and loaded into a microfluidic device. Simultaneous analysis of the two reporter strains in the same device excludes variations in media or other environmental ${ }_{80}$ conditions, and thus allows for a direct comparison of the stress responses. To distinguish between the two cell types, a nuclear marker (Hta2-CFP) was included in the Msn2-FL-GFP strain. Ten stage positions where recorded, and at each position brightfield, CFP, FITC (GFP), Cy3 (mCherry) and Cy5 images were ${ }_{85}$ acquired with a 2 min interval for one hour (Suppl. Figure 6). As shown in Figure 4C, a histogram of the CFP intensity clearly showed two modes; one centred at 200 counts and the other one around 1'500 counts. With a threshold set at 750 counts, there are 57 cells identified that are considered CFP positive and hence 90 contain the Msn2-FL-GFP plasmid (solid blue line), whereas there are 52 cells below the threshold considered to bear the Msn2-NLS-GFP plasmid (dashed red line). The cells were exposed to three different media: a synthetic control medium (SD), a medium lacking glucose and a rich medium supplemented 95 with $0.4 \mathrm{M} \mathrm{NaCl}$. Each medium also contained a slightly different amount of a marker dye (Alexa680-dextran 12-20 $\mu \mathrm{g} / \mathrm{ml}$ ). This dye allowed to identify the different media and thus to verify the proper switching of the flows in the microfluidic device (Figure 4A), and it was also used to segment the cells in the image. Cells 100 were imaged in SD medium for $10 \mathrm{~min}$ then shifted to glucose free medium for $10 \mathrm{~min}$. After a recovery phase of $10 \mathrm{~min}$ in SD medium, cell were subjected to the high osmolarity medium for $10 \mathrm{~min}$, and then finally returned to SD medium. To follow nuclear translocation of Msn2 and Hog1, a secondary object 105 named HiIntPix was defined based on the $30 \%$ brightest pixels of the cell, which allows precise quantification of the dynamics of nuclear relocation by focusing the analysis on pixels belonging to the cytoplasm and the nucleus while eliminating contribution from the vacuole. The change in Hog 1 localization was quantified 110 by measuring the average intensity of the 20 highest pixels in this object (Figure 4B). As expected, Hog1 localization was not affected by glucose starvation but strongly responded to osmotic stress. In contrast, Msn2 relocation behaviour was strikingly different. To quantify these events, we measured the normalized 115 standard deviation of the HiIntPix, a value low when the protein is uniformly distributed across the cell, but that increases when a 
fraction of Msn2 accumulates in the nucleus. Under conditions of glucose starvation or osmotic shock, the Msn2-FL-GFP construct relocalized to the nucleus resulting in a strong increase in the normalized standard deviation. In contrast, Msn2-NLS-GFP only 5 accumulated in the nucleus upon glucose starvation, demonstrating the distinct regulation of this biosensor. Moreover, the Msn2-NLS-GFP signal decreased during the osmotic shock, probably because cell-shrinking results in a higher average fluorescent intensity.

\section{MAPK nuclear relocation}

Although different strategies can be employed to quantify nuclear relocation, the most reliable and accurate method relies on a nuclear marker. Without a proper nuclear stain, it is impossible to distinguish whether lack of nuclear accumulation in a given cell 15 is absent because the signalling cascade is not active or because the nucleus is simply out of focus. In addition, thanks to a nuclear marker the measurements become more quantitative, since the total nuclear pool of a given protein can be monitored. To quantify nuclear relocation, it has proven advantageous to define 20 the ratio between the average intensity in the nucleus and in a cytoplasmic region around the nucleus. To this end, a secondary object up to 4 pixels away but not touching the nucleus was selected and termed AroundNucleus (Suppl. Figure 7). This procedure minimized experimental artefacts such as bleaching, 25 protein expression or shrinking in osmotic stress experiments.

Since nuclear relocation of $\operatorname{Hog} 1^{23,26}$ and several mammalian MAP kinases ${ }^{27}$ provides an invaluable tool to quantify signalling networks, we wanted to verify whether other yeast MAP kinases display a similar behaviour. We therefore tagged the mating MAP 30 kinase Fus3, and the cell wall integrity MAP kinase Mpk1 with GFP in a strain containing a histone fused to mCherry (Hta2). As a positive control, we used a strain expressing Hog1-GFP. The samples were imaged with variable time intervals ranging from 2 to 10 minutes at five different positions with GFP and RFP filter 35 sets. As expected, Hog1-GFP displayed a marked nuclear relocation upon treatment of the cells with $0.4 \mathrm{M} \mathrm{NaCl}$, which was reliably quantified by measuring the ratio between the nuclear and cytoplasmic intensities as a function of time (Figure $5 \mathrm{~A}$ ). This transient nuclear accumulation lasted roughly $30 \mathrm{~min}$, 40 which is consistent with previous measurements of Hog1 kinase activity based on phospho-specific antibodies ${ }^{22}$. In contrast to Hog1, Fus3 is slightly enriched in the nucleus under basal conditions, but the ratio between the nuclear and cytoplasmic intensities remained constant after activation of the pathway by

${ }_{45}$ pheromone addition (Figure 5B). However, Fus3 levels were significantly increased upon pathway activation and the intensity of Fus3-GFP in the cytoplasm and in the nucleus more than triples one hour after induction (Suppl. Figure 8). Together, these data suggest that Fus 3 rapidly shuttles between the cytoplasm by 50 a mechanism that is independent of its activity state ${ }^{28}$.

Finally, the cell wall integrity pathway can be activated by a wide range of stresses ${ }^{29}$, including temperature changes, chemical insults or osmolarity changes in the medium. Interestingly, the nuclear concentration of Mpk1 has been shown to vary depending 55 on the temperature ${ }^{30}$, such that at low temperatures, Mpk1 is inactive but mostly nuclear, while at higher temperatures it is predominantly cytoplasmic. We therefore tested whether hypoosmotic conditions would result in a change in the cytoplasmic and nuclear concentrations of Mpk1. To this end, cells were 60 grown in $1 \mathrm{M}$ sorbitol at $30^{\circ}$ and $\mathrm{H}_{2} \mathrm{O}$ was added to the media to induce the pathway. However, under those conditions, we could not detect a significant change in the ratio of Mpk1 between nucleus and cytoplasm, implying that Mpk1 activation does not trigger its relocation. Taken together, these results demonstrate ${ }_{65}$ that among the three yeast MAP kinases only Hog1 activity correlates with nuclear translocation, and this readout can therefore not be used to monitor activity of the Mpk1 and Fus3 pathways.

\section{Conclusions}

70 The image analysis platform described here provides a versatile and expandable data acquisition and analysis platform that allows measuring a large variety of signalling events in single cells. When coupled to microfluidic devices, this platform is ideally suited to automate temporal and spatial quantification of 75 signalling networks in single cells. The interface based on the Filemaker database offers a convenient entry method to define the experimental parameters, and the integrated image analysis routines written in Matlab allow more advanced users to modify and add new functions to optimize and expand the available 80 quantification methods.

We have used this analysis platform to accurately quantify protein expression dynamics in multiple channels and characterize the nature of expression noise. Fast signaling dynamics can be best quantified by protein relocation assays, and 85 we have integrated such outputs by quantifying the relocation of well-known proteins like the stress responsive transcription factor Msn2 and the MAPK Hog1. Moreover, we have used the described methods to analyse the nuclear / cytoplasmic partitioning of the MAP kinases Fus3 and Mpk1 upon activation 90 by pheromones and hypo-osmotic stress, respectively. While we have focused this report on the measurement of protein expression and nuclear translocation, the developed scripts are well suited to routinely measure many other protein relocation events.

95

\section{Materials and Methods}

\section{Yeast culture}

All experiments were performed with yeast from W303 background. Yeast strains and plasmids are listed in ${ }_{100}$ Supplementary Tables 7 and 8 . The cells were grown overnight in synthetic medium (SD) to saturation. The cells were diluted to $\mathrm{OD}_{600} 0.05$ in the morning and grown for at least four hours before the start of the experiment. For the experiments in well slides, (Fig 3 and 5), the cells were diluted to $\mathrm{OD}_{600} 0.025$, and ${ }_{05}$ briefly sonicated $(2 \mathrm{x} 45 \mathrm{~s})$ before $200 \mu \mathrm{l}$ of cells were added to a well pre-coated with ConcanavalinA (17-0450-01, GE Healthcare). Selected wells of a 96-well-plates (MGB096-1-2LG, Matrical Bioscience) were coated with a filtered solution of Concanavalin A in PBS $(0.5 \mathrm{mg} / \mathrm{ml})$ for $30 \mathrm{~min}$ and rinsed with ${ }_{110} \mathrm{H}_{2} \mathrm{O}$. To stimulate the cells, $100 \mu \mathrm{l}$ of inducing solution was added to the well (Figure 3 and $5 \mathrm{~A}: 1.2 \mathrm{M} \mathrm{NaCl}$ in SD, Figure 5B $300 \mathrm{nM}$ alpha-factor in $\mathrm{SD}$ and Figure $5 \mathrm{C}$ cells were grown in SD $+1 \mathrm{M}$ sorbitol and $100 \mu 1 \mathrm{H}_{2} \mathrm{O}$ was added to induce hypo-osmotic 
stress).

For the microfluidic experiment (Figure 4) the cells were diluted to $\mathrm{OD}_{600} 0.01$, briefly sonicated and $200 \mu$ l of culture was loaded in the chip (Y04C, CellASIC Corp). Synthetic medium without ${ }_{5}$ glucose $(+16 \mu \mathrm{g} / \mathrm{ml}$ Alexa680-Dextran (D34681, Invitrogen)) was loaded in wells 1 and 4, SD medium $(+20 \mu \mathrm{g} / \mathrm{ml}$ Alexa680Dextran) in wells 2 and 5 and $\mathrm{SD}+0.4 \mathrm{M} \mathrm{NaCl}(+12 \mu \mathrm{g} / \mathrm{ml}$ Alexa680-Dextran) in wells 3 and 6 . The pressure was set to 3 psi resulting in an estimated flow rate of $7.5 \mu 1 / \mathrm{hr}$. The flow

10 surrounding the cells, coming either from wells 1 and 4, 2 and 5 or 3 and 6 , was switched by a dedicated control software (ONIX, CellASIC Corp.).

\section{Microscopy}

Images were acquired on a fully-automated inverted epi-

is fluorescence microscopes (Ti-Eclipse, Nikon) controlled by micro-manager ${ }^{12}$ in an incubation chamber set at $30^{\circ} \mathrm{C}$, with $40 \mathrm{X}$ oil objective and appropriate excitation and emission filters. A motorized XY-stage allowed recording multiple fields of view at every time point. Images were recorded at fixed time intervals 20 between 2 and 15 minutes.

Image Analysis

All the images were analysed with the YeastQuant platform. The Filemaker database (FileMaker, version 10 or 11) and the Matlab routines (The Mathworks, R2010a or above) are available at:

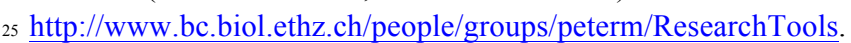
The raw images of the experiments presented in this report, as well as the result from the analysis generated by YeastQuant are available to serve as a training set. A more detailed description of the platform and the installation process can be found in the ${ }_{30}$ Supplementary Online Material.

\section{Acknowledgments}

We thank P. Horvath and members of the Peter laboratory for helpful discussions, and A. Smith for critical reading of the manuscript. This work was supported by UNICELLSYS, the ${ }_{35}$ SystemsX.ch (RTD projects LiverX and YeastX) and the Competence Center for "Systems Physiology and Metabolic Disease" (CC-SPMD). Research in the Peter laboratory is funded by the European Research Counsel, the Swiss National Science Foundation and the ETHZ.

\section{${ }_{40}$ Notes and references}

ETH-Zurich, Institute of Biochemistry, Schafmattstr. 18, CH-8093 Zurich, Switzerland; E-mail: serge.pelet@bc.biol.ethz.ch and matthias.peter@bc.biol.ethz.ch

$\dagger$ Electronic Supplementary Information (ESI) available: Supplementary

45 Text, 7 Supplementary Tables and 12 Supplementary Figures. See DOI: $10.1039 / \mathrm{b} 000000 \mathrm{x} /$

The authors declare to have no competing interests

Author's Contribution: SP wrote the program, performed the experiments and analyzed the data. RD, SSL, FvD and MP provided yeast strains and

50 plasmids, and helped design the experiments. SP and MP drafted the final manuscript, which was read and approved by all authors.

1. J. E. Ferrell and E. M. Machleder Science 1998, 280, 895-898

2. R. Y. Tsien Annual Review of Biochemistry 1998, 67, 509-544

55 3. N. C. Shaner, P. A. Steinbach, and R. Y. Tsien Nature Methods 2005, 2, 905-909

4. A. Colman-Lerner, A. Gordon, E. Serra, T. Chin, O. Resnekov, D. Endy, C. G. Pesce, and R. Brent Nature 2005, 437, 699-706

5. L. Cai, C. K. Dalal, and M. B. Elowitz Nature 2008, 455, 485-
S. Pelet, F. Rudolf, M. Nadal-Ribelles, E. de Nadal, F. Posas, and M. Peter Science 2011, 332, 732-735

7. Lorentzen, A. Kinkhabwala, O. Rocks, N. Vartak, and P. I. H. Bastiaens Science Signaling 2010, 3, ra68-ra68

658 B. Neumann, T. Walter, J.-K. Hériché, J. Bulkescher, H. Erfle, C. Conrad, P. Rogers, I. Poser, M. Held, U. Liebel, C. Cetin, F. Sieckmann, G. Pau, R. Kabbe, A. Wünsche, V. Satagopam, M. H. A. Schmitz, C. Chapuis, D. W. Gerlich, R. Schneider, R. Eils, W. Huber, J.-M. Peters, A. A. Hyman, R. Durbin, R. Pepperkok, and J. Ellenberg Nature 2010, 464, 721-727

10. A. E. Carpenter, T. R. Jones, M. R. Lamprecht, C. Clarke, I. H. Kang, O. Friman, D. A. Guertin, J. H. Chang, R. A. Lindquist, J. Moffat, P. Golland, and D. M. Sabatini Genome biology 2006, 7, R100

11. A. Chernomoretz, A. Bush, R. Yu, A. Gordon, and A. ColmanLerner Current protocols in molecular biology 2008, Chapter 14, Unit 14.18

12. A. Edelstein, N. Amodaj, K. Hoover, R. Vale, and N. Stuurman $80 \quad$ Current protocols in molecular biology 2010, Chapter 14, Unit14.20

13. F. Meyer Signal Processing 1994, 38, 113-125

14. D. H. Ballard Pattern Recognition 1981, 13, 111-122

15. G. Charvin, F. R. Cross, E. D. Siggia, and D. Lew PLoS ONE

$85 \quad 2008, \mathbf{3}$, e 1468

16. P. J. Lee, N. C. Helman, W. A. Lim, and P. J. Hung BioTechniques 2008, 44, 91-95

17. M. B. Elowitz, A. J. Levine, E. D. Siggia, and P. S. Swain Science 2002, 297, 1183-1186

90 18. G. Mas, E. de Nadal, R. Dechant, M. L. R. de la Concepción, C. Logie, S. Jimeno-González, S. Chávez, G. Ammerer, and F. Posas The EMBO journal 2009, 28, 326-336

19. B. G. Reid and G. C. Flynn Biochemistry 1997, 36, 6786-6791

20. M. S. Cyert The Journal of biological chemistry 2001, 276, 95 20805-20808

21. R. C. Yu, C. G. Pesce, A. Colman-Lerner, L. Lok, D. Pincus, E. Serra, M. Holl, K. Benjamin, A. Gordon, and R. Brent Nature 2008, 456, 755-761

22. V. Reiser, H. Ruis, and G. Ammerer Molecular biology of the $100 \quad$ cell $1999,10,1147-1161$

23. P. Hersen, M. N. McClean, L. Mahadevan, and S. Ramanathan Proceedings of the National Academy of Sciences of the United States of America 2008, 105, 7165-7170

24. W. Görner, E. Durchschlag, M. T. Martinez-Pastor, F. Estruch, 105 G. Ammerer, B. Hamilton, H. Ruis, and C. Schüller Genes \& Development 1998, 12, 586-597

25. W. Görner, E. Durchschlag, J. Wolf, E. L. Brown, G. Ammerer, H. Ruis, and C. Schüller The EMBO journal 2002, 21, 135-144

$26 . \quad$ J. T. Mettetal, D. Muzzey, C. Gómez-Uribe, and A. van

$110 \quad$ Oudenaarden Science 2008, 319, 482-484

27. A. G. Turjanski, J. P. Vaqué, and J. S. Gutkind Oncogene 2007, 26, 3240-3253

28. F. van Drogen, V. M. Stucke, G. Jorritsma, and M. Peter Nature cell biology 2001, 3, 1051-1059

$11529 . \quad$ D. E. Levin Microbiology and Molecular Biology Reviews 2005, 69, 262-291

30. Y. Kamada, U. S. Jung, J. Piotrowski, and D. E. Levin Genes \& Development 1995, 9, 1559-1571 
Tables

Table 1. Segmentation methods

\begin{tabular}{llll}
\hline & Requirements & Advantages & Disadvantages \\
\hline $\begin{array}{llll}\text { Cell } \\
\text { Fluorescence }\end{array}$ & $\begin{array}{l}\text { Cytoplasmic fluorescent marker } \\
\text { 1 Fluorescence image }\end{array}$ & Simple & No sub-cellular structure \\
Media & $\begin{array}{l}\text { Fluorescent media } \\
\text { Fluorescence }\end{array}$ & $\begin{array}{l}\text { No cellular fluorescent } \\
\text { marker used for } \\
\text { segmentation }\end{array}$ & Works only in microfluidics \\
& $\begin{array}{l}\text { Nuclear and Cytoplasmic } \\
\text { fluorescent markers }\end{array}$ & $\begin{array}{l}\text { Definition of nuclear and } \\
\text { cytoplasm objects }\end{array}$ & $\begin{array}{l}\text { Takes up two fluorescent } \\
\text { channels }\end{array}$ \\
& $\begin{array}{l}\text { 2 Fluorescence images } \\
\text { Nucleus Cell }\end{array}$ & $\begin{array}{l}\text { No fluorescence } \\
\text { illumination }\end{array}$ & No sub-cellular structure \\
\hline
\end{tabular}




\section{Figure Legend}

Figure 1. Schematic representation of YeastQuant image analysis platform. The microscopy images form a 4D data set. The user 5 enters the description of the experiment in a Filemaker database, and selects both the proper analysis to be performed on these images and the segmentation parameters depending on the analysis routine and experimental parameters such as objective magnification and binning. This information is loaded into Matlab, which carries out the automated image analysis. The individual single cell measurements are then exported, and the user can visualize these for the whole cell population or for individually selected cells.

10 Figure 2. Segmentation routines implemented by the platform. (A) Cellular intensity segmentation for whole cell detection. (B) Fluorescent media segmentation. Individual cells are recognized based on the intensity of a fluorescent dye mixed with the media surrounding the cell. (C) Combination of nuclear and cytoplasmic fluorescent tags allows defining a cellular and nuclear object for each cell. (D) Bright field segmentation for whole cell detection is based on two bright-field images measured at two different focal planes. (E) Definition from secondary objects based on a primary object and an intensity image. A secondary object (HiIntPix, blue) can be 15 defined by selecting, for example, the $30 \%$ of the pixels with the highest intensity for each cell. Another secondary object (Media, red) can be defined by geometrical parameters for pixels surrounding each cell.

Figure 3. Stochastic protein expression upon mild hyper-osmotic shock. (A) Cells bearing the pSTL1-qCFP and pSTL1-qV expression reporters were subjected to $0.1 \mathrm{M}$ (red), $0.15 \mathrm{M}$ (blue) and $0.2 \mathrm{M}$ (green) $\mathrm{NaCl}$. The average fluorescence intensity in the 20 yellow channel and the standard deviation of more than 200 cells is plotted. (B) Scatter plot of the difference between final (2hrs) and initial fluorescence in the YFP channel and CFP channel upon treatment with $0.15 \mathrm{M} \mathrm{NaCl}$. (C and D) Single cell traces of four cells selected in the scatter plot (B) labelled 1 to 4 displaying the change in CFP (C) and YFP (D) intensity as function of time.

Figure 4. Quantification of Msn2 localization in different media. Cells bearing a Hog1-mCherry and Hta2-CFP tags were transformed 25 with a full-length Msn2-GFP plasmid (solid blue line), while cells bearing only the Hog1-mCherry where transformed with an Msn2NLS-GFP (dashed red line). These two strains were mixed and imaged under rich (SD), glucose starvation and osmotic stress conditions. (A) The flows were visualized by different concentrations of marker dye in the medium. Cells were imaged for 60 min in a microfluidic flow chamber, with the following media: $10 \mathrm{~min}$ in SD, $10 \mathrm{~min} \mathrm{~S}$ without glucose (light blue), $10 \mathrm{~min} \mathrm{SD}, 10 \mathrm{~min} \mathrm{SD} \mathrm{+} 0.4 \mathrm{M} \mathrm{NaCl}$ (light green) and $20 \mathrm{~min}$ in SD as visualized by the change in Cy5 intensity. (B) Quantification of the Hog1-mCherry nuclear shuttling 30 based on the change in the average intensity of the 20 brightest pixels belonging to the HiIntPix object. This intensity increases upon addition of $\mathrm{NaCl}$ to the medium (light green). (C) The two strains were differentiated based on their relative intensity in the CFP channel allowing to separate the Msn2-FL-GFP cells (solid blue line) or the Msn2-NLS-GFP cells (dashed red line). (D) Msn2 shuttling quantified by the normalized standard deviation of the GFP signal in the HiIntPix object. Relocation of the full-length allele occurs both under starvation (light blue) and $\mathrm{NaCl}$ stress (light green), while the Msn2-NLS-GFP fragment relocates only under glucose starvation 35 conditions (light blue). The error bars in (A, B and D) represent the standard error of the mean of more than 50 cells.

Figure 5. MAPK nuclear to cytoplasmic relocation upon pathway stimulation. (A) Quantification of Hog1-GFP Hta2-mCherry cells upon hyper-osmotic stress. The relocation of the MAPK to the nucleus is quantified by measuring the ratio of nuclear over peri-nuclear intensity as function of time. (B) Quantification as in (A) of Fus3-GFP, Hta2-mCherry cells upon alpha-factor stimulation. Cells 40 significantly induce the expression of the MAPK but no difference in the ratio of the nuclear over cytoplasmic pool can be detected. (C) Quantification as in (A) of Mpk1-GFP Hta2-mCherry cells upon hypo-osmotic stress. No change in protein localization or expression is detected. All images were acquired with the same exposure time $(200 \mathrm{~ms})$ but scaled linearly between 220 and 1000 counts in (A), 200 and 2500 in (B) and 200 and 1000 in (C). The red and blue curves in (B) and (C) represent the quantification of two independent timelapse movies. The error bars represent the standard error of the mean of 60 to 200 cells depending on the experiment.

45 


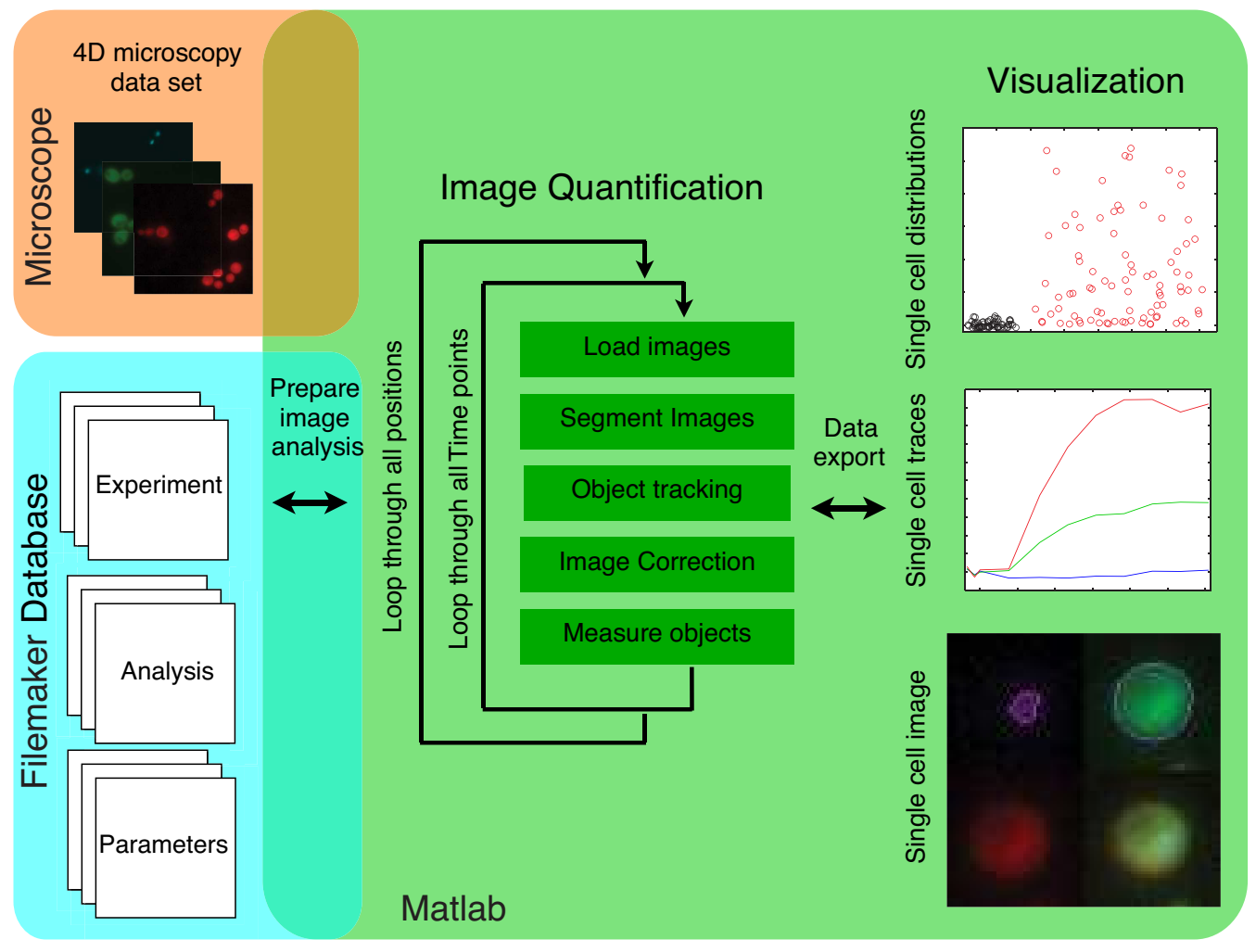

Fig.1 
Input Image
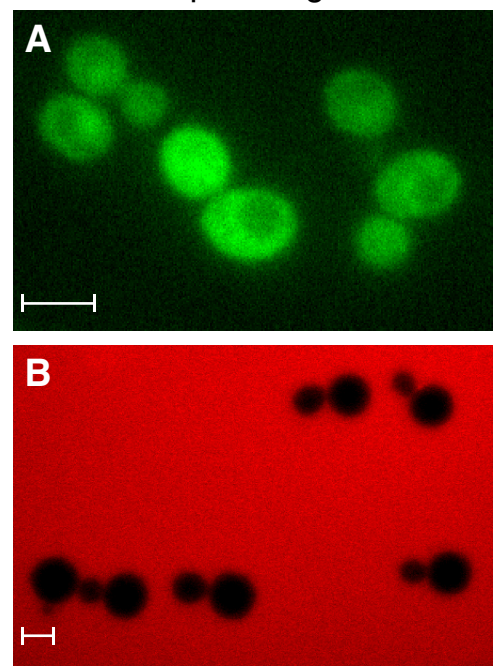

Input Image I

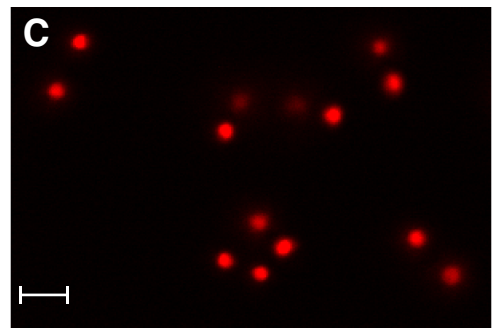

D

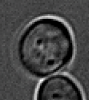

(3)

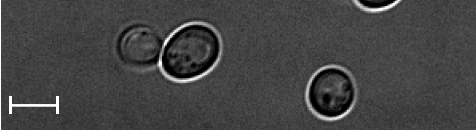

Primary Object

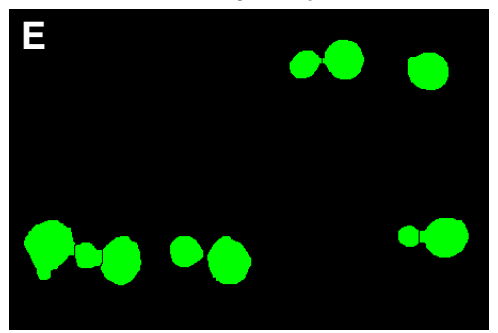

Input Image II
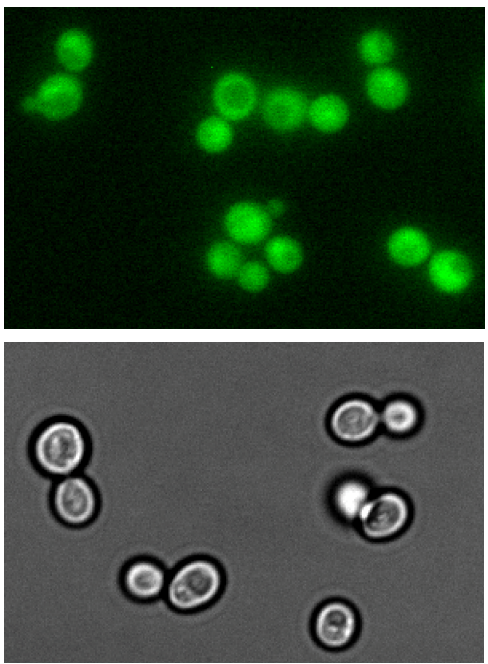

Intensity Image

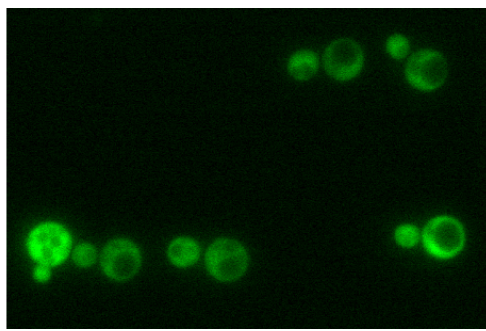

Segmented Objects
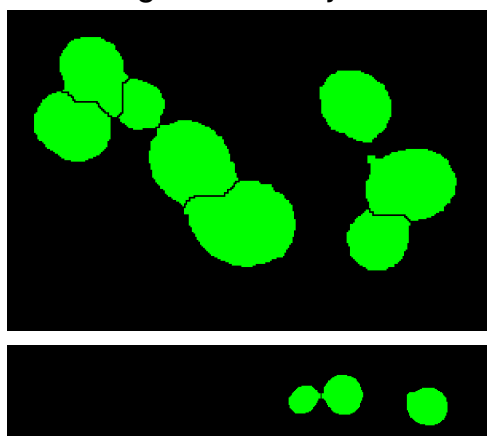

$\infty \infty 0^{\circ} 0$

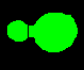

Segmented Objects
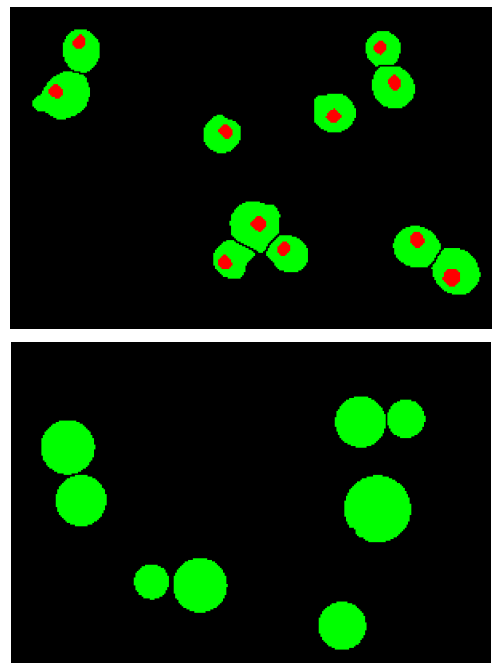

Secondary Objects

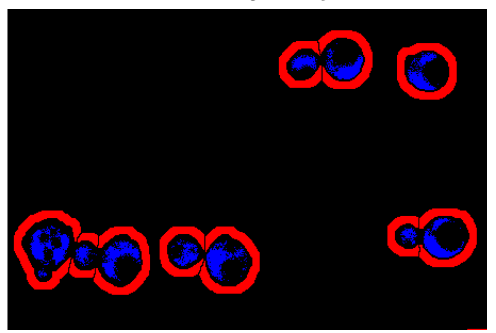

Fig.2 
A

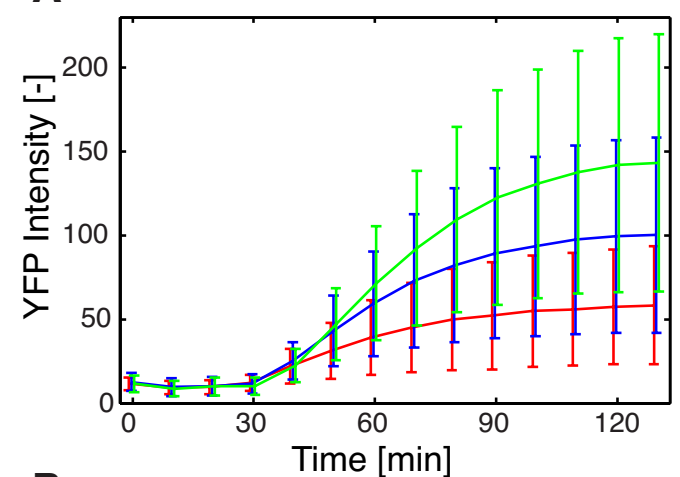

\section{B}

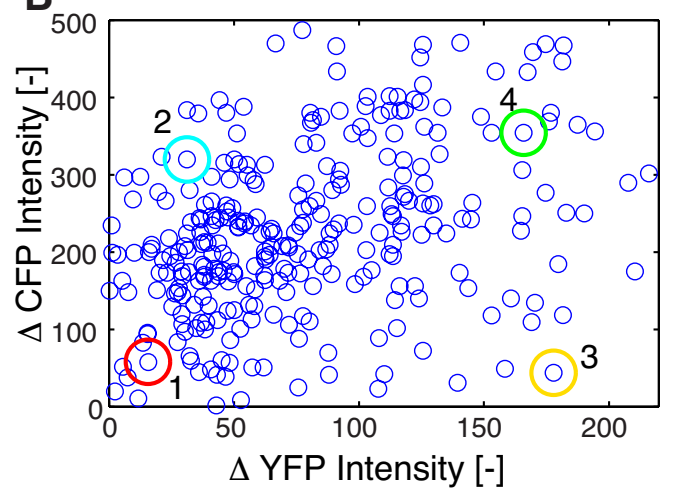

C

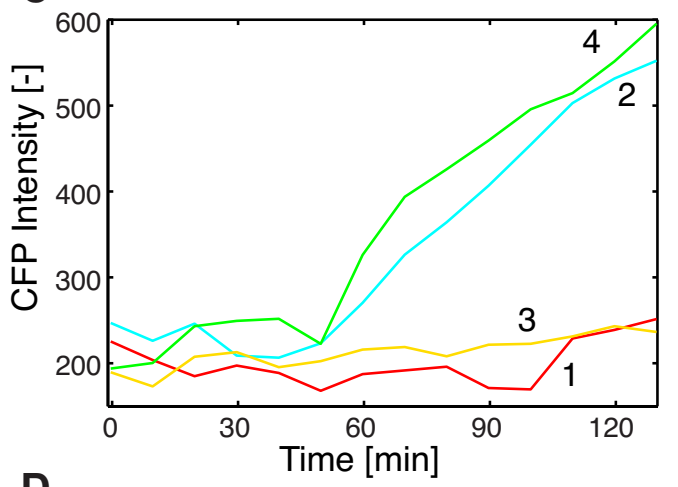

D

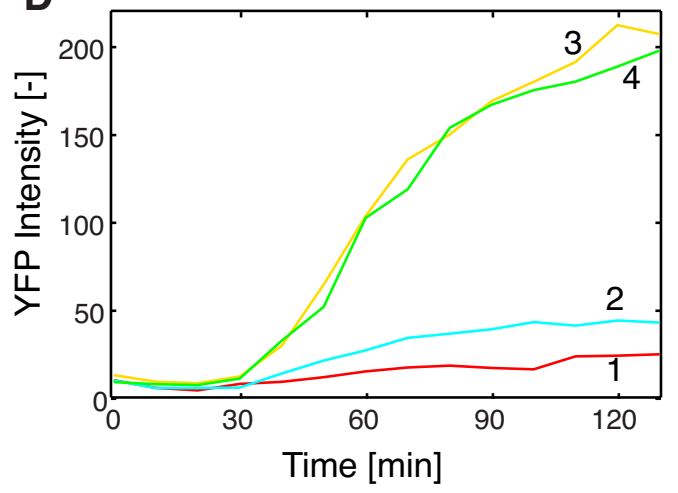

Fig.3 

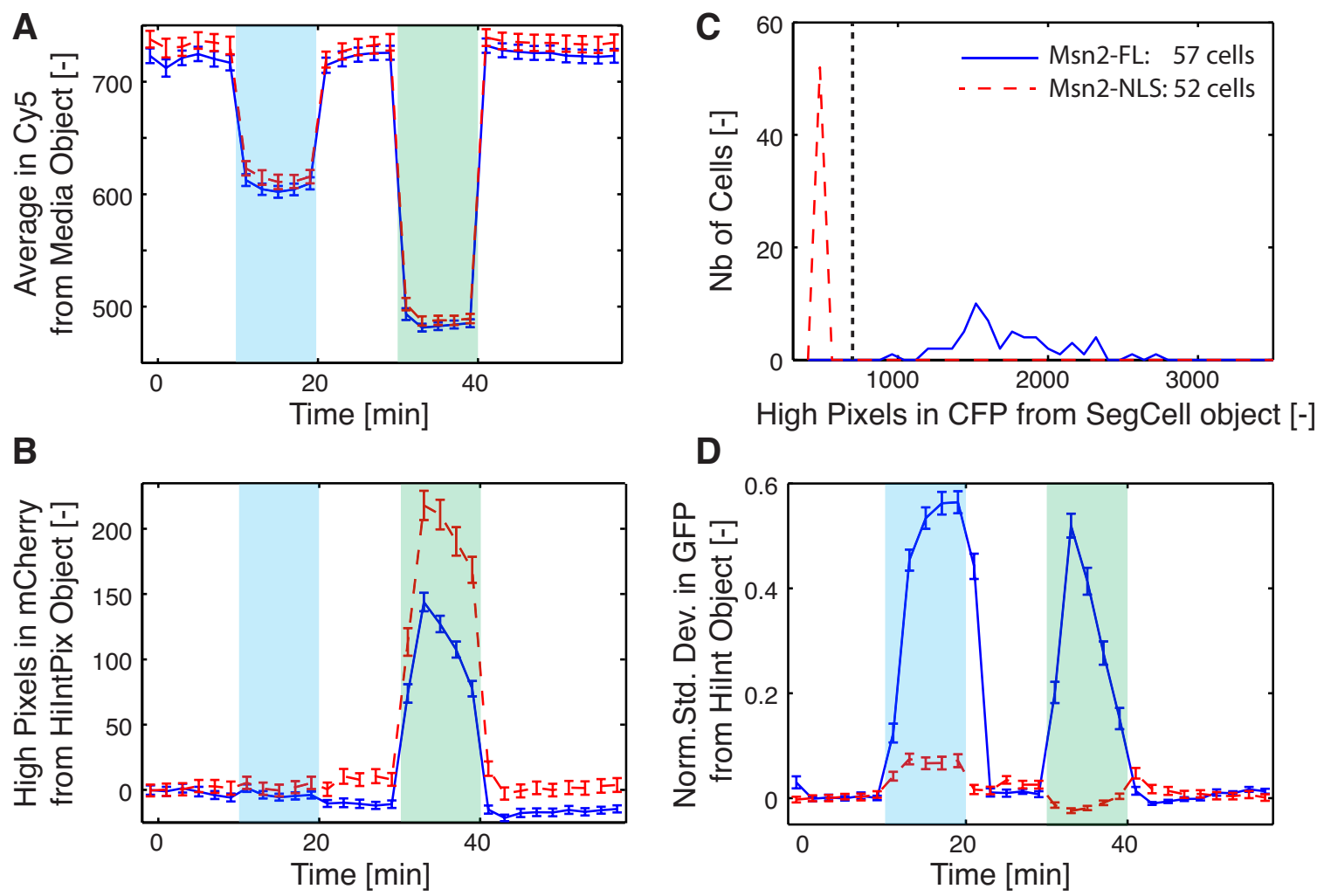

Fig.4 

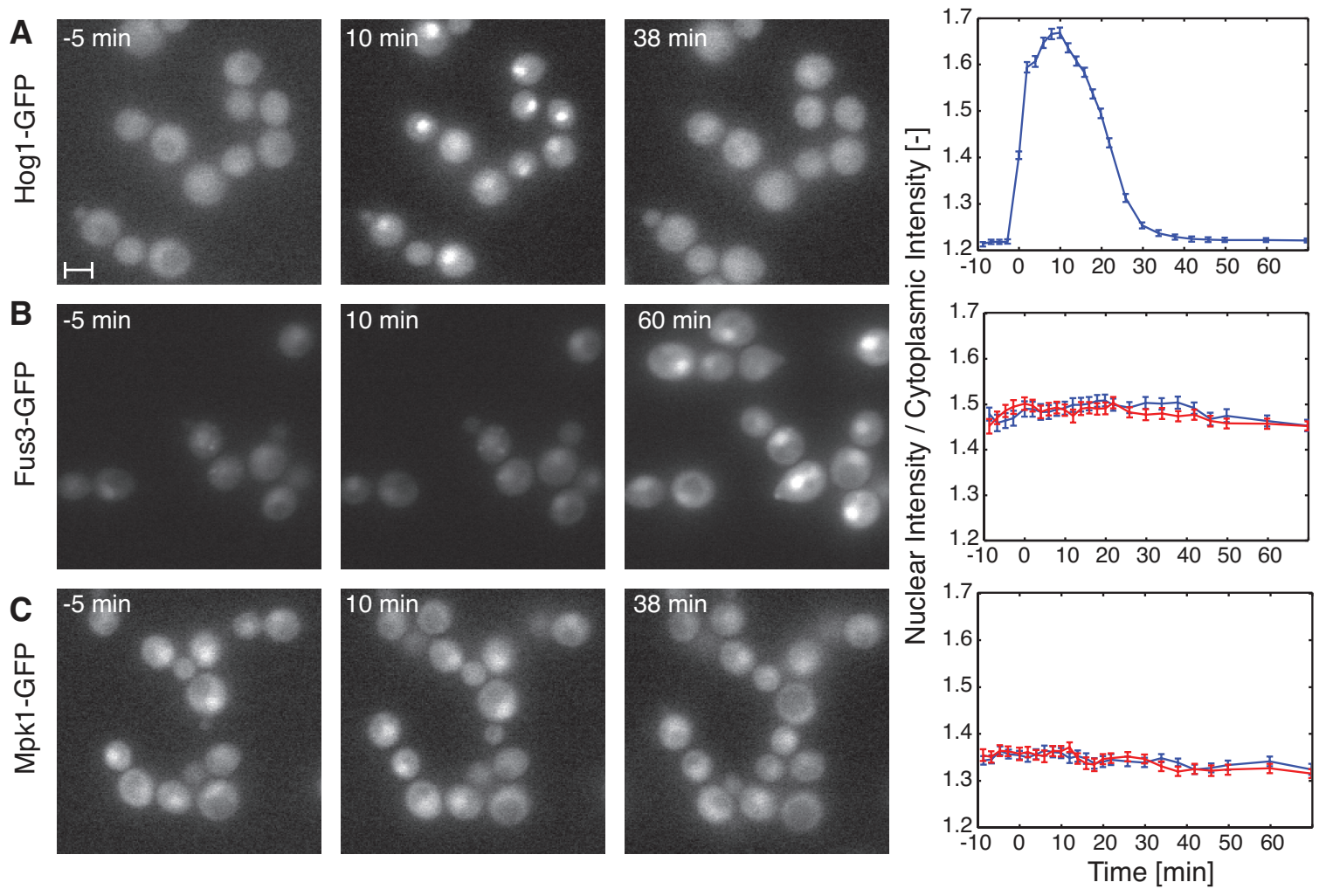

Fig.5 